\title{
New method for numerical simulation of a nonstationary potential flow of incompressible fluid with a free surface
}

\author{
Vladimir E. Zakharov ${ }^{\mathrm{a}, \mathrm{b}, 1}$, Alexander I. Dyachenko ${ }^{\mathrm{a}, *}$, Oleg A. Vasilyev ${ }^{\mathrm{a}}$ \\ a Landau Institute for Theoretical Physics, 2 Kosygin str., Moscow, 117334, Russia \\ ${ }^{\mathrm{b}}$ Department of Mathematics, University of Arizona, Tucson, AZ 857201, USA
}

Received 12 November 2001; received in revised form 22 February 2002; accepted 1 March 2002

\begin{abstract}
New method for numerical simulation of potential flows with a free surface of two-dimensional fluid, based on combination of the conformal mapping and Fourier Transform is proposed. The method is efficient for study of strongly nonlinear effects in gravity waves including wave breaking and formation of rogue waves. (C) 2002 Éditions scientifiques et médicales Elsevier SAS. All rights reserved.
\end{abstract}

\section{Introduction}

The problem of an analytic and numeric description of a non-stationary potential flow in a fluid with a free surface is one of the most fundamental in Hydrodynamics. At the moment several different approaches to solution of this problem are elaborated. A survey of the methods can be found, for example, in [1]. We do not plan to give in this article even a brief review of the methods. We just mention that they can be divided, roughly speaking, in two groups. Some authors assume that nonlinearity is weak and use expansions in powers of the surface slope. In the most advanced works belonging to this direction, they use the Hamiltonian formalism [2]. The most important advantage of this approach is the opportunity to implement such efficient numerical algorithm as the fast Fourier transform. It makes possible to use a very fine mesh and resolve as much as thousands or even dozen of thousands Fourier modes. The obvious disadvantage of this approach is impossibility to describe the most interesting physical processes such as wave breaking (where surface becomes multi-valued function of coordinates) and formation of the freak waves. These processes are essentially nonlinear. Another group of methods (surface integral methods) uses the exact hydrodynamic equation and can in principle be applied for description of the strongly nonlinear phenomena, see, for instance, [3]. But these methods are usually incompatible with the Fourier formalism and allow just a relatively poor resolution.

In this article we offer a completely new approach to analytic and numeric study of a nonstationary flow of the ideal noncompressible fluid with a free surface. It is based on the equations obtained in [4,5]. To our opinion this approach combines the advantages of the existing approaches, being free of these disadvantages. The basic idea of this new method is reformulation of the exact Euler equations describing a potential flow of a fluid with a free surface by the "Hilbert-differential" equations just with a polynomial (cubic) nonlinearity. The new form of the exact equations makes possible to implement the Fourier method even more efficiently than for the approximate equation. So the new method is implemented for periodic boundary conditions.

As a result, we can achieve a very high resolution in description of completely nonlinear phenomena such as wave-breaking and formation of freak waves. So far, we performed only first starting series of numerical experiments. The results are really

\footnotetext{
* Correspondence and reprints.

E-mail addresses: zakharov@itp.ac.ru (V.E. Zakharov), alexd@landau.ac.ru (A.I. Dyachenko).

1 Waves and Solitons Ltd.
} 
impressive. For instance, we clearly observed formation of freak waves as a result of development of the modulational instability of weakly-nonlinear Stokes waves.

\section{Basic equations}

We study a fluid of the infinite depth, occupying the area

$-\infty<y<\eta(x, t)$.

The flow is irrotational, hence

$$
V=\nabla \phi .
$$

The condition of incompressibility $\operatorname{div} V=0$ implies that the velocity potential $\phi$ satisfies the Laplace equation

$$
\triangle \phi=0 .
$$

In the absence of an external pressure, the boundary conditions imposed on $\phi$ and boundary itself are

$$
\frac{\partial \phi}{\partial t}+\frac{1}{2}|\nabla \phi|^{2}+g \eta=0, \quad \frac{\partial \eta}{\partial t}+\eta_{x} \phi_{x}=\phi_{y},
$$

and $\partial \phi / \partial y=0$ at $y \rightarrow-\infty$. The total energy of the system is

$$
\mathcal{H}=\frac{1}{2} \int_{-\infty}^{\infty} \mathrm{d} x \int_{-\infty}^{\eta(x, t)}|\nabla \phi|^{2} \mathrm{~d} y+\frac{g}{2} \int_{-\infty}^{\infty} \eta^{2}(x, t) \mathrm{d} x
$$

$g$ is gravity acceleration. The system is Hamiltonian [6,7] and can be rewritten as follows:

$$
\frac{\partial \eta}{\partial t}=\frac{\delta \mathcal{H}}{\delta \psi}, \quad \frac{\partial \psi}{\partial t}=-\frac{\delta \mathcal{H}}{\delta \eta} .
$$

Here $\psi=\left.\phi\right|_{z=\eta}$ and $\eta$ are canonically conjugated variables. Let us perform the conformal mapping of the domain

$$
-\infty<x<\infty, \quad-\infty<y<\eta(x, t),
$$

filled with fluid, to the half-plane

$$
-\infty<u<\infty, \quad-\infty<v<0 .
$$

After the transform, the shape of the surface $\eta(x, t)$ can be presented in a parametric form

$$
y=y(u, t), \quad x=x(u, t)=u+\tilde{x}(u, t),
$$

here $\tilde{x}(u, t)$ and $y(u, t)$ are related through Hilbert transformation

$$
y=\widehat{H} \tilde{x}, \quad \tilde{x}=-\widehat{H} y, \quad \widehat{H}^{2}=-1 \quad \text { and } \quad \widehat{H}(f(u))=P \cdot V \cdot \frac{1}{\pi} \int_{-\infty}^{\infty} \frac{f\left(u^{\prime}\right) \mathrm{d} u^{\prime}}{u^{\prime}-u} .
$$

After the conformal mapping $\phi(x, y, t) \rightarrow \phi(u, v, t), \psi(x, t) \rightarrow \psi(u, t)$. It was shown in [4] (see also [8,9]), that $y(u, t)$ and $\psi(u, t)$ obey the following system of equations:

$$
\begin{aligned}
y_{t} & =\left(y_{u} \widehat{H}-x_{u}\right) \frac{\widehat{H} \psi_{u}}{J}, \\
\psi_{t} & =-\frac{\psi_{u}^{2}+\widehat{H} \psi_{u}^{2}}{2 J}+\widehat{H}\left(\frac{\widehat{H} \psi_{u}}{J}\right) \psi_{u}+\frac{\widehat{H} \psi_{u}}{J} \widehat{H} \psi_{u}-g y .
\end{aligned}
$$

Here $J$ is the Jacobian of the mapping:

$$
J=x_{u}^{2}+y_{u}^{2}=1+2 \tilde{x}_{u}+\tilde{x}_{u}^{2}+y_{u}^{2} .
$$

Equations (2.4) and (2.5) can be written in the complex form. Let $z=x+\mathrm{i} y, \Phi=\psi+\mathrm{i} \widehat{H} \psi$ are functions analytic in the lower half-plane. They satisfy the equations 


$$
\begin{aligned}
z_{t} & =\mathrm{i} U z_{u}, \\
\Phi_{t} & =\mathrm{i} U \Phi_{u}-B+\mathrm{i} g(z-u) .
\end{aligned}
$$

Here $U$ is a complex transport velocity:

$$
U=\widehat{P}\left(\frac{-\widehat{H} \psi_{u}}{\left|z_{u}\right|^{2}}\right)
$$

and

$$
B=\widehat{P}\left(\frac{\left|\Phi_{u}\right|^{2}}{\left|z_{u}\right|^{2}}\right) .
$$

In (2.9) and (2.10), $\widehat{P}$ is the projector operator generating a function which is analytical in the lower half-plane, $\widehat{P}(f)=$ $\frac{1}{2}(1+\mathrm{i} \widehat{H}) f$.

It occurs (see [5]) that Eqs. (2.7)-(2.8) can be simplified just by changing variables. Indeed, let us introduce, instead of $z(w, t)$ and $\Phi(w, t)$, another functions $R(w, t)$ and $V(w, t)$ in a following way:

$$
R=\frac{1}{z_{w}}, \quad \Phi_{w}=-\mathrm{i} V z_{w}
$$

( $V$ is just i $\partial \Phi / \partial z$, i.e. complex velocity). Note, that because $z(w, t)$ is a conformal mapping, its derivative does exist in the lower half-plane and does not have zeroes there. Thus function $R(w, t)$ is analytic in the lower half-plane and has the following boundary condition:

$$
R(w, t) \rightarrow 1, \quad|w| \rightarrow \infty, \operatorname{Im}(w) \leqslant 0 .
$$

It is obvious that the boundary condition for $V$ is

$$
V(w, t) \rightarrow 0, \quad|w| \rightarrow \infty, \operatorname{Im}(w) \leqslant 0 .
$$

Then equations for these analytic functions acquire a very nice form:

$$
\begin{aligned}
& R_{t}=\mathrm{i}\left(U R^{\prime}-U^{\prime} R\right), \\
& V_{t}=\mathrm{i}\left(U V^{\prime}-R B^{\prime}\right)+g(R-1) .
\end{aligned}
$$

Here (see [5])

$$
B=\widehat{P}^{\prime}(V \bar{V}), \quad U=\widehat{P}(V \bar{R}+\bar{V} R) .
$$

Equations (2.12)-(2.13) are exact and completely equivalent to the initial "traditional" system of equations for the free surface potential flow (2.1). But system (2.12)-(2.13) is much more convenient for analytical and numerical study than the "traditional" system (2.1) or Hamiltonian system (2.3). Indeed, one cannot express $\mathcal{H}$ explicitly in terms of the "natural" variables $\psi$ and $\eta$. At the best one can present $\mathcal{H}$ as an infinite series in powers of $\eta$. As a result, the equations in the "natural" variables are presented by the infinite series as well. This is a big obstacle on the way of their numerical simulation. On the contrary, the new motion equations (2.12)-(2.13) are just polynomial (cubic) in terms of the new variables $R, V$. This circumstance makes possible a more efficient implementation of the fast Fourier transform to solution of the new exact equations.

It should be mention that all three equivalent systems of equations, (2.4)-(2.5), (2.7)-(2.8), (2.12)-(2.13), describing dynamics of free surface in conformal variables, are not PDE. They are "Hilbert-differential" equations including, together with derivatives with respect to $u$,

$$
f \rightarrow \frac{\partial f}{\partial u}
$$

the operation of the Hilbert transformation

$$
f \rightarrow \widehat{H} f \text {. }
$$

From the analytical view-point these two operations are completely different. But from the numerical view-point both operation are similar. Indeed, in terms of Fourier transform, operation (2.14) means

$$
f_{k} \rightarrow \mathrm{i} k f_{k}
$$

while operation (2.15) means

$$
f_{k} \rightarrow \mathrm{i} \operatorname{sign}(k) f_{k} \text {. }
$$

From the computational view-point both these operations are of same level of difficulty. It should be mentioned here that Hilbert transformation "corresponds to" the contour (or surface) integration in the surface integral methods. Of course, Hilbert transformation is much easier to implement numerically. 


\section{Constants of motion, forcing and dissipation}

Dynamic equations describing a fluid with a free surface have natural constants of motion: mass of fluid $M$, horizontal momentum $P_{x}$ and energy $\mathcal{H}$. A serious advantage of the conformal approach is an opportunity to express all of these quantities as integrals over their local densities, which can be expressed explicitly in terms of $z$ and $\Phi$. Indeed, deviation of the mass of fluid from its equilibrium value can be expressed as follows:

$$
\begin{aligned}
& M=\int_{-\infty}^{\infty} m \mathrm{~d} u, \quad m=y x_{u}=\frac{1}{2 \mathrm{i}}\left(z \bar{z}_{u}-z_{u} \bar{z}\right), \\
& P_{x}=\int_{-\infty}^{\infty} p_{x} \mathrm{~d} u, \quad p_{x}=\psi y_{u}=\frac{1}{2 \mathrm{i}} \psi\left(z_{u}-\bar{z}_{u}\right), \\
& \mathcal{H}=\int_{-\infty}^{\infty} w \mathrm{~d} u, \quad w=w_{T}+w_{U},
\end{aligned}
$$

$w_{T}$ is the density of kinetic energy,

$$
w_{T}=-\frac{1}{2} \psi \widehat{H} \psi_{u}=\frac{\mathrm{i}}{8}\left(\Phi \bar{\Phi}_{u}-\bar{\Phi} \Phi_{u}\right),
$$

and $w_{U}$ is the density of potential energy,

$$
w_{U}=\frac{g}{2} y^{2} x_{u}=-\frac{g}{16}(z-\bar{z})^{2}\left(z_{u}+\bar{z}_{u}\right) .
$$

Apparently $w_{T}>0$ and $w_{U}>0$.

Expressions of $m, p$ and $w$ in terms of $R$ and $V$ are not that elegant. One can obtain these expressions using formulae

$$
z=\int \frac{\mathrm{d} u}{R}, \quad \Phi=-\mathrm{i} \int \frac{V}{R} \mathrm{~d} u .
$$

Formulae (3.16)-(3.19) can be used for the control of the numerical calculations.

In reality any system of surface waves is not conservative, and such effects as viscosity and interaction with wind should be taken into account. A complete consideration of these effects is a difficult problem. But this is due to the forcing because of which one can observe wave breaking in the ocean. In many cases one can take dissipative and forcing effect into consideration by the use of some phenomenological modification of the basic equations. It is convenient to perform a modification in Eqs. (2.7)-(2.8). Equation (2.7) is just the kinematic boundary condition on the surface. It should not be modified. The simplest modification of (2.8) is the replacement

$$
\Phi_{t} \rightarrow \Phi_{t}+\hat{\gamma} \Phi
$$

Here $\hat{\gamma}$ is a linear operator of convolution type.

As far as functions $\Phi$ and $z$ are analytical in the lower half-plane, they can be presented in the form

$$
\Phi(u, t)=\frac{1}{\sqrt{2 \pi}} \int_{0}^{\infty} \Phi_{k} \mathrm{e}^{-\mathrm{i} k u} \mathrm{~d} k, \quad z(u, t)=\frac{1}{\sqrt{2 \pi}} \int_{0}^{\infty} z_{k} \mathrm{e}^{-\mathrm{i} k u} \mathrm{~d} k .
$$

Now $(\hat{\gamma} \Phi)_{k}=\gamma_{k} \Phi_{k}$. Here $\gamma_{k}$ is the image of operator $\hat{\gamma}$ in the Fourier space. The equation for the energy balance reads:

$$
\frac{\mathrm{d} \mathcal{H}}{\mathrm{d} t}=-\frac{1}{4} \int_{0}^{\infty} k \gamma_{k}\left|\Phi_{k}\right|^{2} \mathrm{~d} k
$$

Operator $\hat{\gamma}$ provides "dissipation" for wave numbers where $\gamma_{k}>0$, and "forcing" for those $k$, where $\gamma_{k}<0$.

One should take into account that wave numbers for the conformal variables $u$ and for real coordinate $x$ are different. In the linear approximation $\left|Z_{u}\right|^{2}<1$ and $U=\mathrm{i} \Phi_{u}$. Linearization of Eqs. (2.7)-(2.8) leads to the dispersion relation $\left(\Phi \simeq e^{\mathrm{i} \omega t-\mathrm{i} k u}\right)$

$$
\omega_{k}=\frac{\mathrm{i} \gamma_{k}}{2} \pm \sqrt{g k-\frac{\gamma_{k}^{2}}{4}}, \quad 0<k<\infty
$$


The equivalent to (3.21) modification of system (2.12)-(2.13) is the following:

$$
R_{t}=\mathrm{i}\left(U R^{\prime}-U^{\prime} R\right), \quad V_{t}=\mathrm{i}\left(U V^{\prime}-R B^{\prime}\right)+g(R-1)-R \hat{\gamma}\left(\frac{V}{R}\right) .
$$

One can see that the dissipative term in this system is essentially nonlinear.

\section{Numerical algorithm}

All three versions ((2.4)-(2.5), (2.7)-(2.8), (2.12)-(2.13)) of the exact equations for the free surface dynamics of a fluid are convenient for numerical simulation. They are given on the straight line $u$ and allow use of numerical spectral methods. The last one is a very important feature enabling a simple calculation of Hilbert transformation.

In the non-conservative case the equations

$$
z_{t}=\mathrm{i} U z_{u}, \quad \Phi_{t}+\hat{\gamma} \Phi=\mathrm{i} U \Phi_{u}-B+\mathrm{i} g(z-u)
$$

are most convenient. However, in the pure conservative case one should better use the more simple equations (2.12)-(2.13).

Now we will describe some details of numerical simulation of Eqs. (2.12)-(2.13). We use the fourth-order Runge-Kutta integration scheme to solve numerically the equations in the domain of length $2 \pi$. The actual number of negative Fourier modes was equal to $N=12288$, where the total number of points in the real space was $2 N$. All positive Fourier modes are equal to zero. This is a property of projector operator $\widehat{P}$ in the complex plane. So when the projector operator acts on a complex function, it makes zero all spectral modes with positive wave numbers. Numerically it is easy to do with fast Fourier transform. Zero positive spectral modes prevent aliasing - arising the nonphysical modes coupling from nonlinear terms.

To keep our numerical scheme stable, we need to restrict the time step, $\Delta t<1 / N$. This is due to differential operators in Eqs. (2.12)-(2.13). Another obvious condition is $\Delta t<1 / F$, where $F$ is the maximal value of the right-hand side of the equations. In our calculations we keep $\Delta t<0.5 / N$ and $\Delta t<0.05 / F$.

Conservation of the total energy $\mathcal{H}$ provided accuracy of numerical simulation.

\section{Preliminary results}

We plan to include into the derived equations effects of surface tension and perform massive numerical experiments embracing a vast variety of dynamical and statistical problems including simulation of wave breaking, formation of freak waves, and determination of universal spectra of wave turbulence. In this article we present only the very preliminary results obtained in the framework of this program. In the all cases we use Dyachenko equations (2.12)-(2.13) without any kind of damping and forcing. In all our experiments we use the domain $0<x<2 \pi, 0<u<2 \pi$ with periodic boundary conditions. The total number of harmonics was as much as 12288. We assume gravity acceleration $g=10$. In the first experiment we use the initial data in the form

$$
R(u, t=0)=1+a \exp (-\mathrm{i} u), \quad V(u, t=0)=-\mathrm{i} a \sqrt{g} \exp (-\mathrm{i} u),
$$

with the wave number $k=1$. For $a \ll 1$ these initial data lead to formation of propagating wave of small amplitude:

$$
R(u, t)=1+a \exp (-\mathrm{i} u+\mathrm{i} \sqrt{g} t), \quad V(u, t)=-\mathrm{i} a \sqrt{g} \exp (-\mathrm{i} u+\mathrm{i} \sqrt{g} t) .
$$

However, for large $a$ the propagating wave turns to break. In our experiments, we put $a=0.28$, which is just above threshold. Figures 1-2 demonstrate time evolution of the wave profile and the surface spectrum. The spectra $R(k)$ for corresponding time moments are shown on Fig. 3. One can see the tendency to singularity formation as well as to development of spectral "tails". Calculations were terminated when the absolute value of $R(k)$ reached the level $|R(k)| \sim 10^{-10}$ at the end of spectral interval $N=12288$.

The second experiment demonstrates development of modulational instability and formation of the freak waves. Modulational instability of Stokes waves was discovered independently and almost simultaneously by V.E. Zakharov [6,7] and T.B. Benjamin and J.E. Feir [10]. Nonlinear stage of this instability was not investigated so far.

A Stokes wave is characterized by its steepness $\mu=k a$ ( $k$-wave number, $a$-wave amplitude). If $\mu \ll 1$, the Stokes wave is very close to a linear monochromatic progressive wave. In our experiments we put $\mu=0.1$. It makes possible to neglect a deviation of the Stokes wave from exponential one and choose initial data in a form of superposition of the monochromatic wave with wave number $k=-50$ and the Gaussian random noise:

$$
R(u, t=0)=0.1 \exp (-50 \mathrm{i} u)+\frac{5 \times 10^{-5}}{\sqrt{2 \pi}} \sum_{k=0}^{N} \operatorname{rand}_{1}(k) \exp \left(-\frac{\left(50-k_{S}\right)^{2} u^{2}}{800}-\mathrm{i} k u+2 \pi \operatorname{irand}_{2}(k)\right)
$$




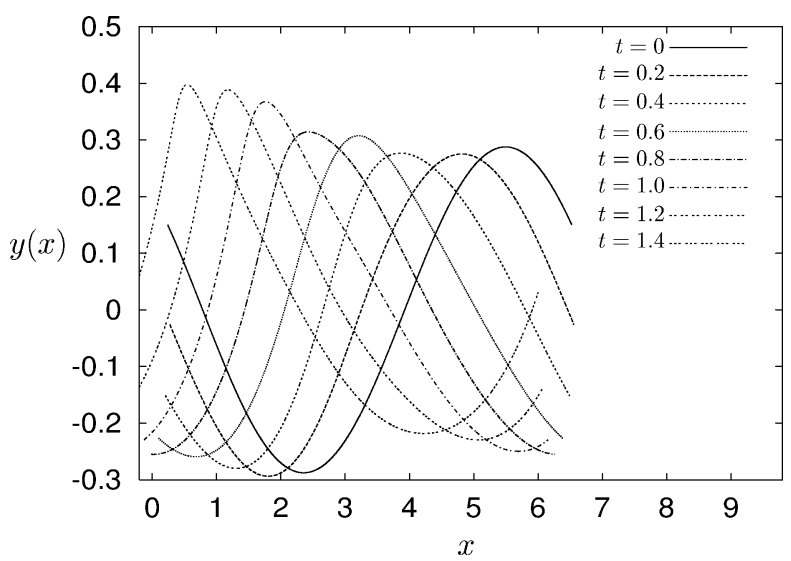

Fig. 1. Wave breaking: the surface of the fluid $y(x)$ at the moments of time $t=0.2-1$. Initial amplitude $a=0.28$.

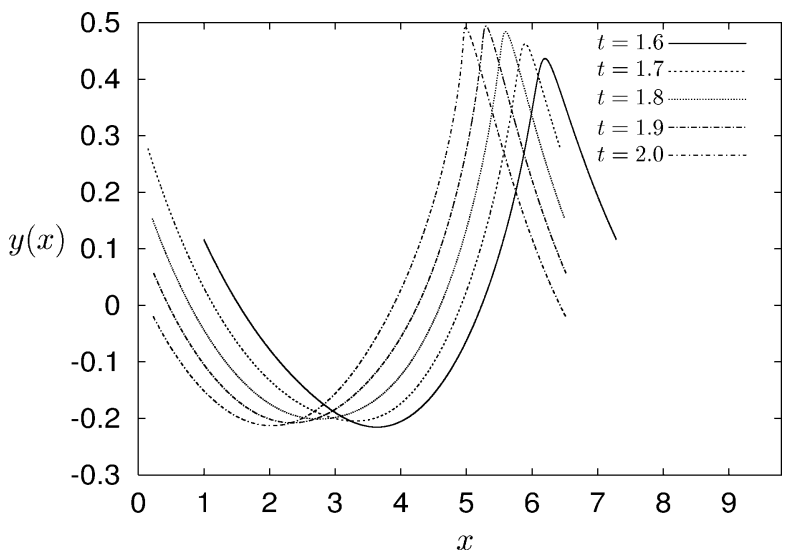

Fig. 2. Wave breaking: the surface of the fluid $y(x)$ at the moments of time $t=1.6-2.0$. Initial amplitude $a=0.28$.

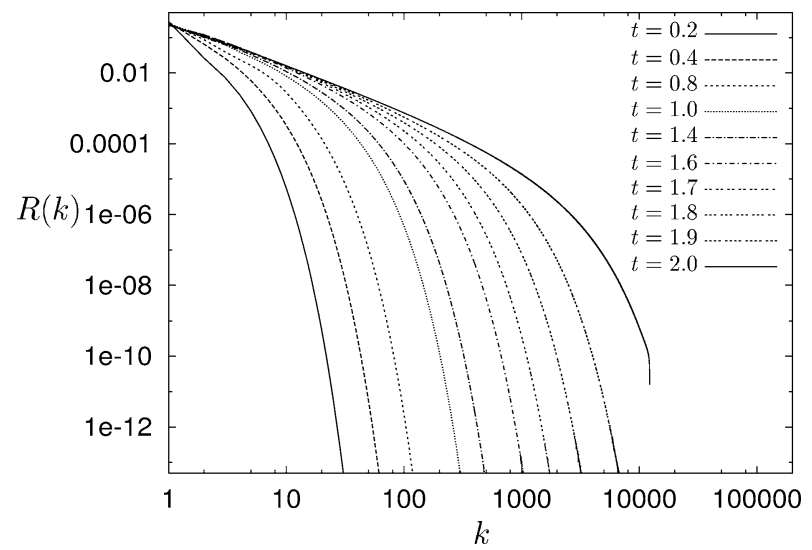

Fig. 3. Wave breaking: the spectra $R(k)$ of the function $R(u)$ at the different moments of time $t$. Initial amplitude $a=0.28$.

$$
\begin{aligned}
V(u, t=0)= & -\mathrm{i} \frac{0.1}{\sqrt{5}} \exp (-50 \mathrm{i} u) \\
& -\frac{5 \times 10^{-5} \mathrm{i}}{\sqrt{2 \pi}} \sum_{k=0}^{N} \frac{\sqrt{10}}{\sqrt{k}} \operatorname{rand}_{1}(k) \exp \left(-\frac{\left(50-k_{s}\right)^{2} u^{2}}{800}-\mathrm{i} k u+2 \pi \mathrm{irand}_{2}(k)\right) .
\end{aligned}
$$




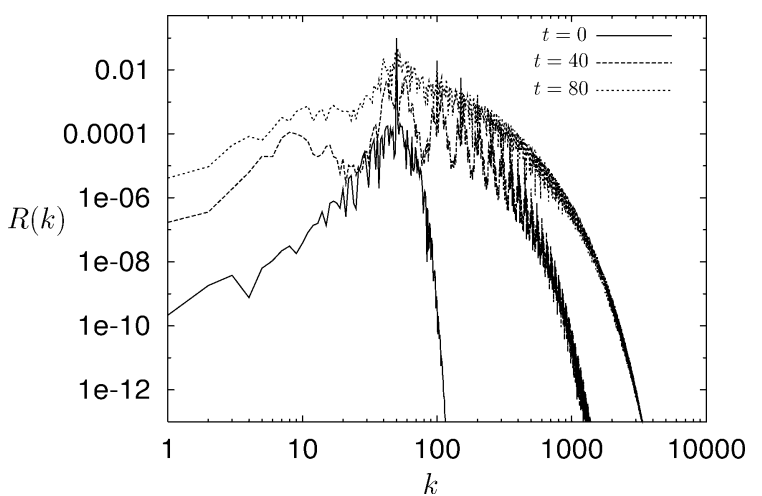

Fig. 4. Development of modulational instability: the spectra $R(k)$ of the function $R(u)$ at the different moments of time $t$.

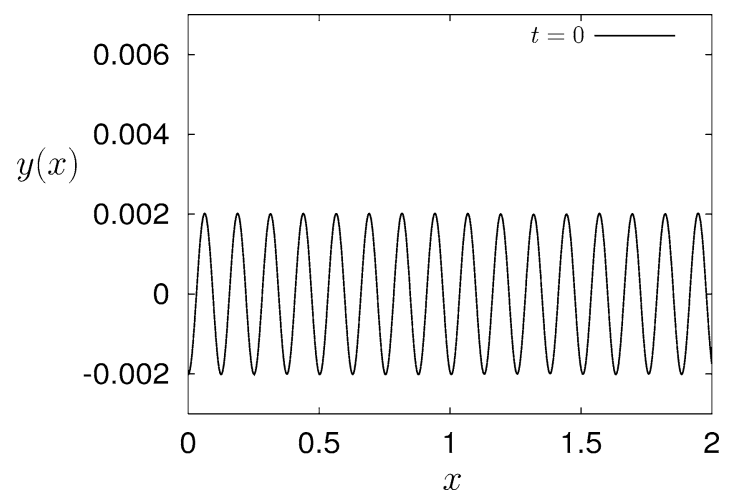

Fig. 5. Development of "freak" waves due to nonlinear interaction: the surface of the fluid for the initial time moment $t=0$.

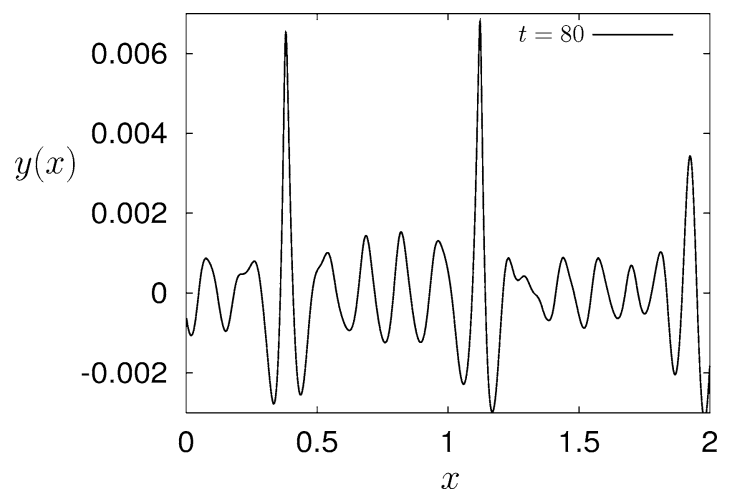

Fig. 6. Development of "freak" waves due to nonlinear interaction: the surface of the fluid for the time moment $t=80$.

The initial spectra of function $R$ is shown on Fig. 4 (its mark is $t=0$ ), and a part of initial surface $y(x)$ is shown on Fig. 5 .

Forcing and dumping are absent in our computations. The total energy remains constant during simulation up to $t=90$. After that the spectra become some wide that one needs to increase the number of Fourier modes, or to stop the simulation.

The initial spectra $(t=0)$ and its evolution $t=40$ and $t=80$ are shown on Fig. 4 . So we can see how smooth continuous spectra develop from single spectral harmonic with a "bit" of noise. The spectral "tail" steady propagates in the area of high wave numbers. This process can be interpreted as formation of singularities on the crests of individual waves, in other words, as onset of wave-breaking. To continue calculations beyond the moment $t=80$, one should include dissipation into calculation.

The most impressive results of our experiments on simulation of nonlinear stage of the modulational instability is the fast formation of "freak waves". Figure 5 presents the initial shape of the fluid surface. One can see, that the initial random noise imposed on the monochromatic wave is very small. Figure 6 presents the shape of the surface in the end of our calculation. 


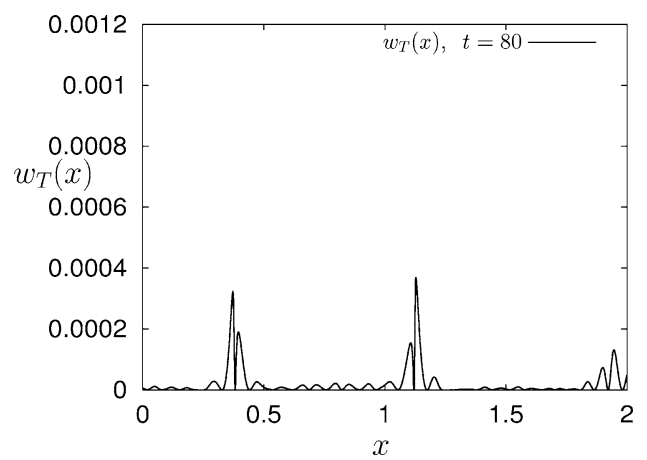

Fig. 7. Development of "freak" waves due to nonlinear interaction: density of the kinetic energy $w_{T}(x)$ a) for the time moment $t=80$.

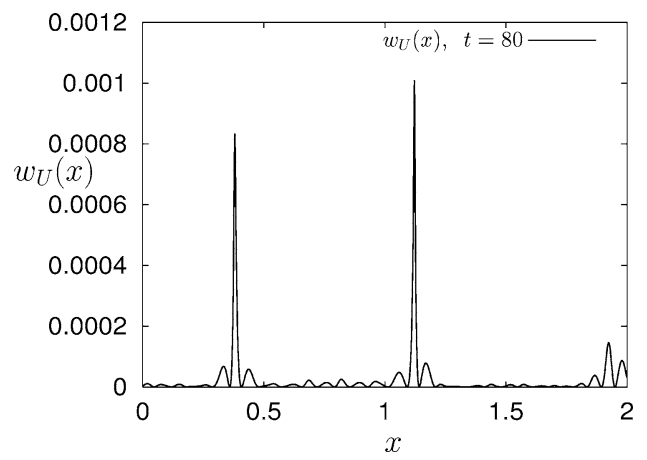

Fig. 8. Development of "freak" waves due to nonlinear interaction: density of the potential energy $w_{U}(x)$ for the time moment $t=80$.

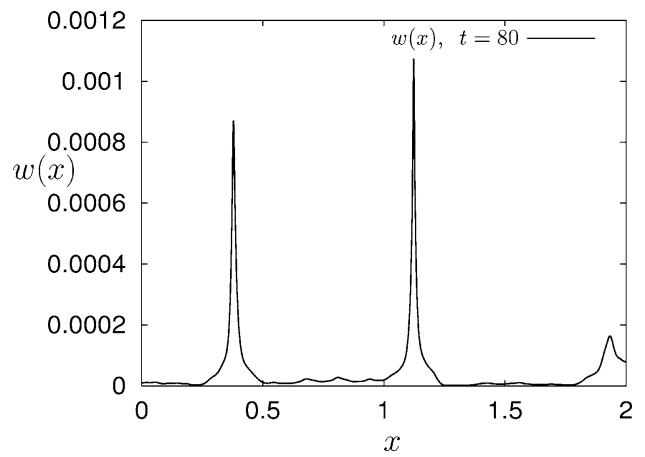

Fig. 9. Development of "freak" waves due to nonlinear interaction: density of the total energy $w(x)$ for the time moment $t=80$.

One can see the formation of "freak" or "rogue" waves with the amplitude exceeding the initial level more than three times. Figures 7-9 which displays the distribution of densities of kinetic, potential and total energy are even more spectacular. One can see that the total energy density in freak waves exceeds the average level almost in two order of magnitudes.

\section{Conclusions}

We developed a very efficient numerical model for numerical simulation of dynamics of an ideal incompressible fluid with a free surface. We will show in our next publication, that the effects of dissipation and surface tension can be included in the algorithm in a very natural way. The new method makes possible to perform massive experiments for a careful study of wave-breaking, interaction of capillary waves and generation of capillary waves by gravity waves. We plan also to perform a systematic study of formation of freak waves including mechanism of their appearance from the "smooth sea". As far as our new algorithm is fast enough, we will be able to study the statistics of freak waves and to find PDF of their generation. 


\section{Acknowledgements}

We are grateful Joint SuperComputer Center (www.jscc.ru) for disposal of computational resources. This research was partially supported by INTAS-96-0413 Grant, by ONR Grant \# N00014-98-1-0070, by DACA 42-00C0044, by RBRF Grant No. 00-01-00929 and by the Grant of Leading Scientific Schools of Russia 00-15-96007.

\section{References}

[1] W. Tsai, D. Yue, Computations of nonlinear free-surface flows, Annu. Rev. Fluid Mech. 28 (1996) 249-278.

[2] W. Craig, C. Sulem, Numerical simulation of gravity waves, J. Comput. Phys. 108 (1993) 73-83.

[3] J.W. Dold, D.H. Peregrine, in: Water-Wave Modulation, 20th International Conference on Coastal Engineering, ASCE, Taipei, Vol. 103, 1986, pp. 163-176.

[4] A.I. Dyachenko, E.A. Kuznetsov, M.D. Spector, V.E. Zakharov, Analytical description of the free surface dynamics of an ideal fluid (canonical and formalism and conformal mapping), Phys. Lett. A 221 (1996) 73-79.

[5] A.I. Dyachenko, On the dynamics of an ideal fluid with a free surface, Dokl. Math. 63 (1) (2001) 115-118; Translated from: Dokl. Akad. Nauk 376 (1) (2001) 27-29.

[6] V.E. Zakharov, The instability of waves in nonlinear dispersive media, Zh. Eksp. Teor. Fiz. 51 (1956) 1107-1114;

V.E. Zakharov, Sov. Phys. JETP (1967) 24.

[7] V.E. Zakharov, Stability of periodic waves of finite amplitude on the surface of a deep fluid, J. Appl. Mech. Tech. Phys. 27 (1968) 86-94.

[8] A.I. Dyachenko, V.E. Zakharov, Toward an integrable model of deep water, Phys. Lett. A 221 (1996) 80-84.

[9] V.E. Zakharov, A.I. Dyachenko, High-Jacobian approximation in the free surface dynamics of an ideal fluid, Physica D 98 (1996) $652-664$.

[10] T.B. Benjamin, J.E. Feir, The disintegration of wave trains on deep water, Part 1. Theory, J. Fluid Mech. 27 (1967) 417. 